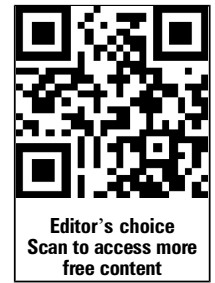

${ }^{1}$ Department of Physiotherapy, La Trobe University, Melbourne, Victoria, Australia ${ }^{2}$ Department of Medicine, Nursing and Health Sciences, Monash University, Melbourne, Victoria, Australia

\section{Correspondence to}

Dr Tania Pizzari,

Department of Physiotherapy, La Trobe University, Bundoora, Mebourne 3086, Victoria, Australia;

T.Pizzari@latrobe.edu.au

Accepted 5 July 2013 Published Online First 5 August 2013

\title{
The predictive validity of a single leg bridge test for hamstring injuries in Australian Rules Football Players
}

\author{
Grant Freckleton, ${ }^{1}$ Jill Cook, ${ }^{2}$ Tania Pizzari ${ }^{1}$
}

\section{ABSTRACT}

Background Hamstring muscle strain injuries (HMSI) are the greatest injury problem in kicking sports such as Australian Rules Football. Reduced hamstring muscle strength is commonly perceived to be a risk factor for hamstring injury; however, evidence is inconclusive. Testing hamstring strength with the hip and knee at functional angles and assessing endurance parameters may be more relevant for examining the risk of hamstring injury.

Objective The primary aim of this prospective study was to examine if reduced hamstring muscle strength assessed with the single leg hamstring bridge (SLHB) was a risk factor for hamstring injury.

Methods Hamstring muscle strength of 482 amateur and semielite players from 16 football clubs, mean age 20.7 (range 16-34 years), was tested during the 2011 preseason. Players were then monitored throughout the 2011 playing season for HMSI.

Results A total of 28 hamstring injuries, 16 right and 12 left, were recorded. Players who sustained a right HMSI during the season had a significantly lower mean right SLHB score $(p=0.029)$, were older $(p=0.002)$ and were more likely to have sustained a past right hamstring injury $(p=0.02)$ or right knee injury $(p=0.035)$. For left-sided hamstring injury, the injured group was more likely to be left leg dominant $(p=0.001)$, older athletes $(p=0.002)$ and there was a trend towards a history of left hamstring injury $(p=0.07)$. Conclusions This study demonstrated a significant deficit in preseason SLHB scores on the right leg of players that subsequently sustained a right-sided hamstring injury. Age, previous knee injury and a history of hamstring injury were other risk factors supported in this study. Low hamstring strength appears to be a risk factor for hamstring injury; however, due to the confounding variables and low injury rate in this study, further studies are required.

\section{INTRODUCTION}

Hamstring muscle strain injuries (HMSI) are the greatest injury problem in Australian Rules Football (ARF). In elite ARF, hamstring injuries have been the most prevalent injury over the past 10 years, with a mean of 21 missed games per club per season and a recurrence rate of $23 \% .{ }^{1}$ Other sports have reported hamstring injuries to be a substantial problem with similar recurrence rates and time-off sport. In the Australian National Rugby League, hamstring injuries accounted for a mean of 8.3 missed games per season. ${ }^{2}$ In English Premier League football, hamstrings were the most prevalent injury, accounting for $12 \%$ of all injuries reported over two seasons. ${ }^{3}$ Similar to elite ARF, a recurrence rate of $17 \%$ was reported for hamstring injuries in elite football, ${ }^{4}$ with a mean of 4 matches missed per injury. ${ }^{5}$ The identification of players at risk for this injury could assist with the implementation of preventative strategies and result in the reduction of injury rates and reduce the impact of this condition on the sporting club and the athlete.

Hamstring injury is a complex and multifactorial problem. A large volume of research has investigated the risk factors for hamstring injury across many different sports with conflicting outcomes. A recent systematic review and meta-analysis of 34 studies $^{6}$ discovered that quadriceps peak torque, older age and previous history of hamstring injury were risk factors for injury. Other parameters such as limb dominance, playing position, ethnicity, ankle dorsiflexion (DF) range of movement (ROM) and previous knee injury had some limited evidence.

Strength is commonly perceived to be a risk factor for hamstring strain; however, evidence is inconclusive. ${ }^{78}$ It is logical that strength and endurance are important and possibly implicated in hamstring injury. ${ }^{9}$ A weak muscle may fatigue earlier during activity and therefore have to work harder than its physiological capacity to maintain performance during high intensity activity. However, this theory has not been supported by studies that examine hamstring muscle strength using isokinetic testing $^{6}$ that predominantly use peak torque as the outcome, rather than endurance parameters. ${ }^{6}{ }^{10}$ Additionally, isokinetic testing may not assess the hamstring muscles in its most susceptible position.

Hamstring injuries during sprinting are proposed to occur during the terminal swing phase of running as a consequence of an eccentric contraction. ${ }^{11} 12$ Testing the hamstring in a more functional capacity similar to terminal swing and assessing endurance parameters may better evaluate strength as a risk factor for injury. Furthermore, the use of isokinetic equipment may be restricted by access and expense. A clinical test that examines hamstring muscle strength and endurance would be more suited for screening purposes.

The single leg hamstring bridge (SLHB) test is a clinical test for hamstring function used in screening programmes at the elite level. ${ }^{13}{ }^{14}$ Such a simple clinical test of hamstring strength with the hip and knee at functional angles could be feasible to examine risk of hamstring injury.

The primary aim of this prospective study was to examine if reduced hamstring muscle strength assessed with the SLHB was a risk factor for hamstring injury in amateur and subelite Australian football players. Secondary aims were to investigate the association of other factors in the literature with conclusive and inconclusive evidence. These 
included age, dominant leg, previous hamstring injury, injury history, occupation and ankle DF ROM.

\section{METHOD}

\section{Participants}

A total of 482 amateur and semielite players, mean age 20.7 (range 16-34 years) from 16 clubs in the Transport Accident Commission cup (under 18 competition), Victorian Football League and amateur football clubs were recruited for this prospective study. Ethics approval was granted by La Trobe University Faculty Human Ethics Committee. All players, and guardians where the player was under 18 years of age, were informed of the procedure and provided written consent.

Assessors attended preseason training sessions of the clubs and all available players training at the time of the assessment were invited to participate during the training session. A mean of 30.1 players were tested per club during the 2011 preseason. Players were excluded if they were younger than 16 or older than 35 years of age, had sustained a hamstring injury less than 6 weeks prior to testing or had a concurrent injury that precluded them from performing the tests. Recent hamstring injury was an exclusion for safety reasons and due to the high recurrence rate during the first 3 weeks post injury. ${ }^{15}$ Athletes older than 35 years were excluded as older age is a significant risk factor for hamstring injury ${ }^{16}$ and could confound the results.

\section{Outcome measures}

The SLHB test protocol used in the present study has previously been shown to be reliable (intratester intraclass correlation coefficient $(\mathrm{ICC})=0.77-0.89$, intertester $\mathrm{ICC}=0.89-0.91) .{ }^{13}$ Players were instructed to lie down on the ground with one heel on a box. The box measuring $60 \mathrm{~cm}$ high was used for all participants (figure 1). The test leg was positioned in approximately $20^{\circ}$ knee flexion. Participants were instructed to cross arms over the chest and push down through the heel to lift their bottom off the ground. Players were advised that the aim of the test was to do as many repetitions as possible until failure. Consistent feedback was provided throughout the procedure to ensure that the correct technique was achieved. It was essential that each trial included the participant touching their bottom onto the ground, without resting, and then extending the hip to $0^{\circ}$. The non-working leg was required to be held stationary in a vertical position to ensure that momentum was not gained by swinging this leg. When the correct form was lost, one warning was given and the test was ceased at the next fault in technique. Repetition maximum was recorded and the test was then

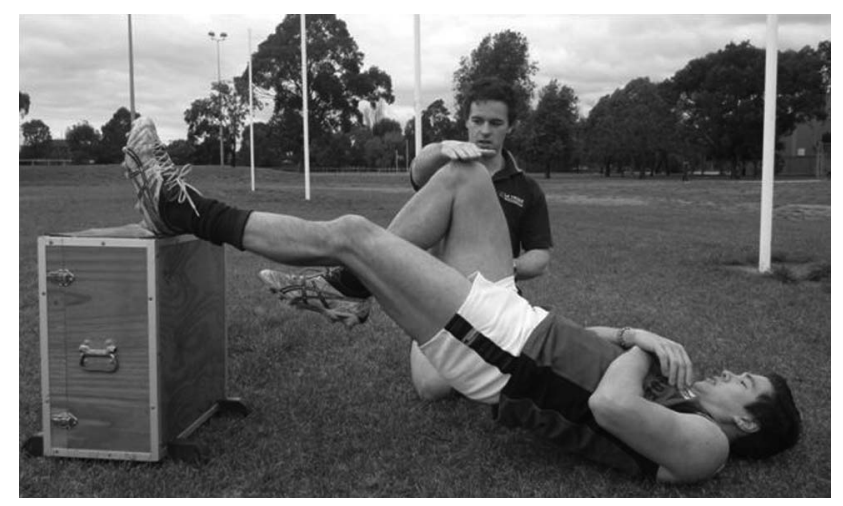

Figure 1 Single leg hamstring bridge. repeated on the opposite leg. The side tested first was alternated between participants.

Ankle DF ROM was measured using a lunge-to-wall test. DF measured using this method has excellent reliability (intratester ICC $=0.98$, intertester ICC $=0.96-0.99) .{ }^{17} 18$

The players also completed a questionnaire that recorded age, occupation, ethnicity, dominant kicking leg, previous hamstring injury, previous knee (ligament) injury and injury history. For the purpose of this study, the dominant leg was the preferred kicking leg of a player, not the stance limb.

\section{Procedures}

Participants were tested by one of the two authors (GF and TP). Players completed the questionnaire and the test procedures were explained. The SLHB was the first test performed and DF ROM followed. Players were then monitored throughout the 2011 playing season for the incidence of a hamstring injury and any recurrences. Follow-up occurred on a weekly basis via phone call and email to participating clubs to determine if any hamstring injuries had been sustained. For the purposes of this investigation, it was required that all injuries were diagnosed by a physiotherapist or a doctor. If a club did not have a qualified physiotherapist or a doctor available, and the player did not seek professional advice, one of the authors (TP) confirmed the diagnosis. Clinical examination for determining hamstring injury is considered to be reliable and accurate. ${ }^{19}$ A hamstring injury was defined as having positive clinical signs and symptoms including the immediate onset of posterior thigh pain, tenderness on palpation, reproduction of pain on stretch of hamstring, reduced straight leg raise ROM and reproduction of pain and reduced strength on resisted active contraction of hamstring muscle group. Players had to be unable to continue playing or training and have missed at least one game due to the hamstring injury. ${ }^{20}$

\section{Data analysis}

Data were analysed using SPSS software package V.18.0 (SPSS Inc, Chicago, Illinois, USA). HMSI were separated into left or right side and analysed independently since there was evidence of performance difference between sides. Univariate analysis using an independent samples $t$ test was used to determine the effect of the leg tested first on SLHB scores. Owing to the unequal group sizes, the Mann-Whitney $U$ test was used to assess the differences between the injured and the uninjured groups for SLHB, age and DF values, and the impact of previous knee or hamstring injury on SLHB scores. $\chi^{2}$ Test was used for categorical information. Spearman's correlation analysed the relationship between age against SLHB scores and the association between occupation and injury. Occupation was defined as sedentary, manual or a mixture of both. Univariate ORs and CIs for hamstring injury were calculated for history of knee injury and previous hamstring injury. ${ }^{21}$ Any variable identified as being associated with hamstring injury in univariate analysis (ie, $\mathrm{p}<1.0)$ was entered into the logistic regression to determine predictive value of tests and combined risk factors. For all tests, a $\mathrm{p}$ value $<0.05$ was considered significant.

\section{RESULTS}

A total of 28 hamstring injuries (16 right and 12 left) were sustained during the playing season (table 1). The leg assessed first for SLHB had the potential to interfere with the opposing leg score due to fatigue; however, there was no significant difference between scores obtained for leg tested first $(p>0.05)$. 
Table 1 Participant characteristics

\begin{tabular}{|c|c|c|c|}
\hline Characteristic & Injured & Uninjured & Total \\
\hline Players (n) & 28 & 454 & 482 \\
\hline $\begin{array}{l}\text { Right leg dominant (n (\% } \\
\text { total)) }\end{array}$ & 19 & 384 & $403(83.6 \%)$ \\
\hline Left leg dominant ( $\mathrm{n}(\%$ total)) & 9 & 70 & $79(16.4 \%)$ \\
\hline Sedentary occupation (n) & 17 & 279 & 296 \\
\hline Manual occupation (n) & 9 & 125 & 134 \\
\hline Mixed occupation (n) & 1 & 31 & 32 \\
\hline $\begin{array}{l}\text { Right SLHB score (reps) (mean } \\
\text { (SD)) }\end{array}$ & $20.31(6.77)$ & $25.98(10.51)$ & $25.79(10.45)$ \\
\hline $\begin{array}{l}\text { Left SLHB score (reps) (mean } \\
\text { (SD)) }\end{array}$ & $27.58(10.52)$ & $26.18(10.15)$ & $26.21(10.15)$ \\
\hline
\end{tabular}

\section{Univariate analysis}

Players who sustained a right hamstring injury during the season had a significantly lower mean right SLHB score compared with uninjured players $(\mathrm{U}(476)=2513, \mathrm{Z}=-2.18, \mathrm{p}=0.029)$. Left SLHB scores for players who sustained a left hamstring injury were not significantly different from uninjured players $(\mathrm{U}(475)$ $=2612, \mathrm{Z}=-0.378, \mathrm{p}=0.705$; table 2).

The mean age of the injured group (left and right legs) was $22.96(\mathrm{SD}=4.85)$, and this was significantly higher than the mean age for uninjured group $(20.58, \mathrm{SD}=3.82, \mathrm{p}=0.002)$. Occupation had no relationship to injury $(p=0.802)$. For rightsided HMSI, there was an association with previous right hamstring injury, older age and previous right knee injury. For leftsided hamstring injury, there was an association with being left leg dominant, older age and a trend towards an association with a previous history of left hamstring injury. Athletes with a history of right HMSI were more than three times likely to have sustained a subsequent right side HMSI (OR $=3.64,95 \%$ CI 1.28 to 10.34). Results were similar for left side injuries $(\mathrm{OR}=3.29,95 \% \mathrm{CI} 0.96$ to 11.25$)$. A history of a right knee injury resulted in an increased risk of a right-sided hamstring injury (OR=3.47, 95\% CI 1.16 to 10.36$)$. Left knee injury was not a significant risk for left hamstring injury $(\mathrm{OR}=0.68,95 \%$ CI 0.09 to 5.42 ).

Asymmetry of SLHB performance between legs was examined for its contribution to hamstring injury. Mean asymmetry between legs was not different between injured players (4.89 reps, $\mathrm{SD}=3.88)$ and uninjured players $(4.58 \mathrm{reps}, \mathrm{SD}=5.12$; $\mathrm{U}$ $(474)=5338, Z=-1.048, p=0.295)$.

\section{Factors influencing SLHB scores}

Players with a history of hamstring strain had reduced SLHB scores (table 3). Players with a history of right knee injury also had reduced SLHB scores on the injured side. Age was negatively correlated to left SLHB score $(r=-0.42, \mathrm{p}<0.001)$ and right SLHB score $(\mathrm{r}=-0.48, \mathrm{p}<0.001)$, highlighting it as a confounder.

\section{Multivariate analysis of risk factors for HMSI}

Previous right knee injury trended towards significance as the largest risk for sustaining a right-sided HMSI (table 4). Left leg dominance was the major risk for sustaining a left side HMSI (table 5).

\section{DISCUSSION}

This prospective study investigated SLHB as a clinical test for predicting hamstring injury and demonstrated a significant deficit in preseason SLHB scores on the right leg of players who sustained a subsequent right-sided injury. The previously identified risk factors of age and a history of hamstring injury were again supported in this study. Previous right knee injury was associated with subsequent right hamstring injury and a preference for left leg kicking was associated with left hamstring injury.

Univariate analysis suggests that the SLHB may be a valuable screening tool. SLHB tests the hamstring in a more functional capacity similar to terminal swing where the hamstring may be at greater risk, ${ }^{11}{ }^{12}$ and assesses endurance parameters. The SLHB is quick and easy to administer with no expensive testing equipment required and allows a large volume of athletes to be tested, although strict adherence to the protocol is required to enhance the predictive validity of the test.

The results of the SLHB as a risk factor were somewhat confounded by age and previous knee injury or hamstring injury, and it was difficult to isolate the contribution of the SLHB in the risk profile. The number of repetitions attained on the SLHB test was negatively correlated with age and there was a significant reduction in the SLHB scores of those players who had sustained either a knee injury or a hamstring injury in the

Table 2 Univariate analysis of variables associated with left and right HMSI

\begin{tabular}{|c|c|c|c|c|}
\hline Variable & Right injured & Left injured & Uninjured & $\mathrm{p}$ Value \\
\hline Right SLHB (reps) (mean (SD)) & $20.31(6.77)$ & - & $25.98(10.51)$ & $0.029+$ \\
\hline Left SLHB (reps) (mean (SD)) & - & $27.58(10.52)$ & $26.18(10.15)$ & $0.705 t$ \\
\hline Previous right HMSI (n) & 6 & - & 66 & $0.02 \ddagger$ \\
\hline Previous left HMSI (n) & - & 4 & 62 & $0.07 \ddagger$ \\
\hline Mean age (years) (mean (SD)) & $23.50(4.47)$ & $22.25(5.23)$ & $20.58(3.82)$ & $0.002^{*} \dagger$ \\
\hline Previous right knee injury $(n)$ & 5 & - & 54 & $0.035 \ddagger$ \\
\hline Previous left knee injury (n) & - & 1 & 55 & $1.00 \ddagger$ \\
\hline Right mean DF ROM (cm) (mean (SD)) & $12.53(4.27)$ & - & $11.69(3.59)$ & $0.339+$ \\
\hline Left mean DF ROM (cm) (mean (SD)) & - & $13.18(4.05)$ & $11.79(3.44)$ & $0.146 \dagger$ \\
\hline Right dominant leg (n) & 14 & 5 & 384 & $1.00 \ddagger$ \\
\hline Left dominant leg $(n)$ & 2 & 7 & 70 & $0.001 \ddagger$ \\
\hline
\end{tabular}


Table 3 SLHB results previous injury versus no history of injury

\begin{tabular}{|c|c|c|c|c|c|c|}
\hline Variable & $\begin{array}{l}\text { Previous ipsilateral } \\
\text { HMSI }\end{array}$ & $\begin{array}{l}\text { No history ipsilateral } \\
\text { HMSI }\end{array}$ & p Value & $\begin{array}{l}\text { Previous ipsilateral knee } \\
\text { injury }\end{array}$ & $\begin{array}{l}\text { No previous ipsilateral knee } \\
\text { injury }\end{array}$ & $p$ Value \\
\hline Left SLHB reps & 22.48 & 26.8 & $0.001^{*}$ & 22.02 & 26.75 & 0.001 * \\
\hline $\begin{array}{l}\text { Right SLHB } \\
\text { reps }\end{array}$ & 21.01 & 26.63 & $<0.001^{*}$ & 22.95 & 26.18 & $0.008^{*}$ \\
\hline
\end{tabular}

past, highlighting the relationship between these variables and their effect on SLHB scores. When all these variables were analysed in a logistic regression, no significant factors were identified highlighting the complex interaction between the variables.

This study had an injury rate of only 5.5\%. This is substantially less than the reported $15 \%$ hamstring injury rate in an elite population of ARF players ${ }^{1}$ and the reported $13.7 \%$ rate in amateur/semielite community level players. ${ }^{22}$ The low injury rate may have reduced the probability of identifying the most important risk factors in the regression analysis. ${ }^{23}$ It is difficult to determine whether this reduced SLHB was a result of the previous strain that has not returned to full strength, or whether the reduced SLHB was the risk factor. The low number of injuries prevented stratification of the population by previous hamstring injury to assess the influence of the SLHB. Similarly, the impact of age on muscle strength and injury exposure confounds the ability to identify the importance of each factor. Logistic regression allows for confounders in the results $^{24}$; however, the numbers in this study were not large enough to accurately discriminate the contribution of the multiple factors involved.

As noted, HMSI is a complex and multifactorial problem. Age and previous hamstring injury are known risk factors for HMSI. $^{3} 61016$ 25-30 Previous right knee injury demonstrated a relationship with right hamstring injury and there is some support for this in the literature. ${ }^{31} 32$ The average SLHB scores and their association with injury are similar to the benchmarks suggested in Hallet (2010). ${ }^{13}$ A score less than 20 is considered poor, 25 average and greater than 30 good. On average, players who sustained a right-sided hamstring injury in this study were close to or below the 'poor' level.

The players who sustained a left HMSI recorded a higher mean SLHB score than the uninjured group. However, these results were due to a relatively small number of left hamstring injuries sustained and the influence of one outlier in the injured group. Only 12 left hamstrings were recorded and more injuries are needed to accurately determine if SLHB is associated with left-sided injury. Similarly, the smaller number of left leg dominant players may have influenced the association between left leg

Table 4 Variables in logistic regression for right HMSI

\begin{tabular}{|c|c|c|c|c|c|}
\hline Variable & B & SE & $\begin{array}{l}\text { Risk } \\
\text { ratio }\end{array}$ & $95 \% \mathrm{Cl}$ & $\begin{array}{l}p \\
\text { Value }\end{array}$ \\
\hline Right SLHB & 0.046 & 0.035 & 1.047 & 0.978 to 1.12 & 0.190 \\
\hline Previous right HMSI & 0.664 & 0.589 & 1.942 & 0.612 to 6.162 & 0.260 \\
\hline $\begin{array}{l}\text { Previous right knee } \\
\text { injury }\end{array}$ & 0.966 & 0.577 & 2.627 & 0.848 to 8.141 & 0.094 \\
\hline Age & -0.099 & 0.067 & 0.906 & 0.794 to 1.033 & 0.140 \\
\hline
\end{tabular}

dominance and left hamstring injury. The multifactorial nature of hamstring injury risk must also be considered a factor in the differing results between sides.

Limitations to this study exist and must be acknowledged. Potential bias existed as all players at each club were not assessed. Attempts were made to test all available players on the evening of testing; however, sampling error as a result of the entire team list not being present at preseason training sessions may have resulted. Completing preseason training has been shown to decrease the number of injuries sustained throughout the playing season. ${ }^{33}$ It is possible that players who did not complete preseason testing are at greater risk of hamstring injury due to poor physical conditioning. Following the off season, sports-specific neural deconditioning, relative muscle weakness and fatigability may make some athletes vulnerable to HMSI throughout the playing season. ${ }^{34}$ The data synthesis method of separating the scores for each leg rather than calculating a combined score for each player could also be questioned. It was decided before data analysis that given the difference in performance issues identified during testing each side should be treated as presenting a separate risk score. The direct relationship between the performance of a muscle and a tear in the same muscle could then potentially be clearer.

Future research is required and the authors suggest the SLHB be tested in an elite ARF population where hamstring injuries are common and entire teams are screened during the preseason. This may allow the extent of confounding variables to be fully evaluated and to identify the relevance of the SLHB.

\section{CONCLUSION}

HMSI is a complex and multifactorial problem. This study demonstrated a significant deficit in preseason SLHB scores on the right leg of players who subsequently sustained a right-sided hamstring injury. Age, previous knee injury and a history of hamstring injury were other risk factors supported in this study. The results of the SLHB as a risk factor are promising; however, due to the confounding variables and low injury rate in this study, it is difficult to isolate the contribution of the SLHB in the risk profile.

Table 5 Variables in logistic regression for left HMSI

\begin{tabular}{lrrlrr}
\hline Variable & B & SE & Risk ratio & $95 \%$ Cl & p Value \\
\hline Dominant leg & 2.159 & 0.613 & 8.664 & 2.604 to 28.827 & $<0.001$ \\
Previous left HMSI & 1.166 & 0.690 & 3.211 & 831 to 12.403 & 0.091 \\
Age & -0.068 & 0.077 & 0.934 & 804 to 1.086 & 0.376 \\
\hline B, regression coefficient; HMSI, hamstring muscle strain injuries.
\end{tabular}




\section{What are the new findings?}

- A reduced preseason single leg hamstring bridge (SLHB) score was identified for players who sustained a right-sided hamstring injury.

- The number of repetitions on the SLHB was negatively correlated with age.

- There was a significant reduction in the SLHB scores of those players who had sustained either a knee injury or hamstring injury in the past.

\section{How might it impact on clinical practice in the near}

future?

- Single leg hamstring bridge (SLHB) test could be used to screen and identify athletes who are potentially at risk of sustaining a hamstring injury.

- SLHB may be used to evaluate the readiness of an athlete to return to sport.

- SLHB could be used to assess the effectiveness of rehabilitation programmes for hamstring injury.

Acknowledgements The authors would like to thank all members of the research team, the injury reporting personnel, the football clubs and all the participants who took part in the study. Professor Cook was supported by the Australian centre for research into sports injury and its prevention, which is one of the International Research Centres for Prevention of Injury and Protection of Athlete Health supported by the International Olympic Committee (IOC).

Contributors All the authors contributed to the development of the research study, data collection, analysis, interpretation of data and writing of this manuscript.

Funding Funding was received from the AFL Research Board.

Competing interests None.

Patient consent Obtained.

Ethics approval La Trobe University Faculty Human Ethics Committee.

Provenance and peer review Not commissioned; externally peer reviewed.

\section{REFERENCES}

1 Orchard J, Seward H. The AFL Injury Report 2010. Australian Football League, 2011.

2 Orchard J. Missed time through injury and injury management at an NRL club. Sport Health 2004;22:11-20.

3 Woods C, Hawkins R, Maltby S, et al. The Football Association Medical Research Programme: an audit of injuries in professional football-analysis of hamstring injuries. Br J Sports Med 2004;38:36-41.

4 Petersen J, Thorborg K, Nielsen M, et al. Acute hamstring injuries in Danish elite football: a 12 month prospective registration study among 374 players. Scand J Med Sci Sports 2010;20:588-92

5 Hawkins RD, Hulse MA, Wilkinson C, et al. The association football medical research programme: an audit of injuries in professional football. $\mathrm{Br}$ I Sports Med 2001;35:43-7.

6 Freckleton G, Pizzari T. Risk factors for hamstring muscle strain injury in sport: a systematic review and meta-analysis. Br J Sports Med 2013;47:351-8.
7 Prior M, Guerin M, Grimmer K. An evidence-based approach to hamstring strain injury: a systematic review of the literature. Sports Health 2009;1:154-64.

8 Foreman T, Addy T, Baker S, et al. Prospective studies into the causation of hamstring injuries in sport: a systematic review. Phys Ther Sport 2006;7:101-9.

9 Opar DA, Williams MD, Shield AJ. Hamstring strain injuries: factors that lead to injury and re-injury. Sports Med 2012;42:209-26.

10 Henderson G, Barnes CA, Portas MD. Factors associated with increased propensity for hamstring injury in English Premier League soccer players. I Sci Med Sport 2010;13:397-402.

11 Schache AG, Wrigley TV, Baker R, et al. Biomechanical response to hamstring muscle strain injury. Gait Posture 2009;29:332-8.

12 Heiderscheit BC, Hoerth DM, Chumanov ES, et al. Identifying the time of occurrence of a hamstring strain injury during treadmill running: a case study. Clin Biomech 2005;20:1072-8.

13 Hallet P. A reliability study examining the inter- and intra-observer reliability of the muscle capacity tests included in the ECB musculoskeletal screening protocol [Masters]. University of Nottingham, 2010.

14 Taylor C, Cook J, Pizzari T, et al. Developing and evaluating a relevant, comprehensive and consistent musculoskeletal protocol for Australian football. Melbourne: Australiasian Football Conference, 2008.

15 Warren P, Gabbe BJ, Schneider-Kolsky M, et al. Clinical predictors of time to return to competition and of recurrence following hamstring strain in elite Australian footballers. Br J Sports Med 2010:44:415-9.

16 Gabbe BJ, Bennell KL, Finch CF. Why are older Australian football players at greater risk of hamstring injury? I Sci Med Sport 2006:9:327-33

17 Bennell $\mathrm{K}$, Talbot $\mathrm{R}$, Wajswelner $\mathrm{H}$, et al. Intra-rater and inter-rater reliability of a weight-bearing lunge measure of ankle dorsiflexion. Aust I Physiother 1998:44:175-80.

18 Dennis RJ, Finch CF, Elliott BC, et al. The reliability of musculoskeletal screening tests used in cricket. Phys Ther Sport 2008;9:25-33.

19 Schneider-Kolsky M, Hoving J, Warren P, et al. A comparison between clinical assessment and magnetic resonance imaging of acute hamstring injuries. Am J Sports Med 2006;34:1008-15.

20 Bennell K, Tully E, Harvey N. Does the toe-touch test predict hamstring injury in Australian Rules footballers? Aust I Physiother 1999;45:103-9.

21 Armitage P, Berry G. Statistical methods in medical research. 3rd edn. Oxford: Blackwell Science, 1994

22 Gabbe B, Finch C, Wajswelner $\mathrm{H}$, et al. Australian football: injury profile at the community level. J Sci Med Sport 2002;5:149-60.

23 Peduzzi $\mathrm{P}$, Concato J, Kemper $\mathrm{E}$, et al. A simulation study of the number of events per variable in logistic regression analysis. J Clin Epidemiol 1996:49:1373-9.

24 Kleinbaum DG, Klein M, Pryor ER. Logistic regression: a self-learning text. Springer Verlag, 2010.

25 Arnason A, Sigurdsson SB, Gudmundsson A, et al. Risk factors for injuries in football. Am J Sports Med 2004:32(1 Suppl 1):5S-16S.

26 Engebretsen $\mathrm{AH}$, Myklebust $\mathrm{G}$, Holme I, et al. Intrinsic risk factors for hamstring injuries among male soccer players: a prospective cohort study. Am I Sports Med 2010;38:1147-53.

27 Gabbe BJ, Bennell KL, Finch CF, et al. Predictors of hamstring injury at the elite level of Australian football. Scand I Med Sci Sports 2006:16:7-13.

28 Gabbe BJ, Finch CF, Bennell KL, et al. Risk factors for hamstring injuries in community level Australian football. Br J Sports Med 2005:39:106-10.

29 Hagglund $\mathrm{M}$, Walden $\mathrm{M}$, Ekstrand J. Previous injury as a risk factor for injury in elite football: a prospective study over two consecutive seasons. $\mathrm{Br}$ I Sports Med 2006:40:767-72

30 Verrall GM, Slavotinek JP, Barnes PG, et al. Clinical risk factor for hamstring muscle strain injury: a prospective study with correlation of injury by magnetic resonance imaging. Br J Sports Med 2001;35:435-40.

31 Koulouris $G$, Connell DA, Brukner $P$, et al. Magnetic resonance imaging parameters for assessing risk of recurrent hamstring injuries in elite athletes. Am I Sports Med 2007:35:1500-6.

32 Verrall GM, Slavotinek JP, Barnes PG, et al. Assessment of physical examination and magnetic resonance imaging findings of hamstring injury as predictors for recurrent injury. I Orthop Sports Phys Ther 2006;36:215.

33 Heidt RS, Sweeterman LM, Carlonas RL, et al. Avoidance of soccer injuries with preseason conditioning. Am J Sports Med 2000;28:659-62.

34 Elliott MCCW, Zarins B, Powell JW, et al. Hamstring muscle strains in professional football players. Am J Sports Med 2011;39:843-50. 